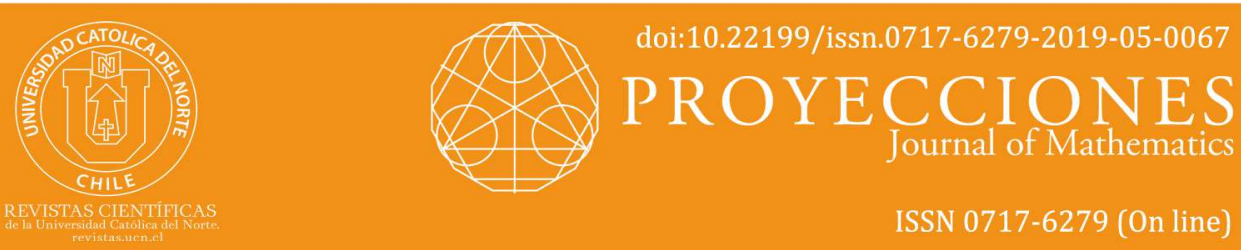

\title{
$\mu$-Statistically convergent function sequences in probabilistic normed linear spaces
}

\author{
Mausumi Sen* (iD) orcid.org/0000-0002-3278-8985 \\ Rupam Haloi* $^{*}$ (iD orcid.org/0000-0003-3788-1414 \\ Binod Chandra Tripathy ${ }^{* * *}$ (iD) orcid.org/0000-0002-0738-652X \\ *National Institute of Technology, Dept. of Mathematics, Silchar, AS, India. \\ $\nabla$ senmausumi@gmail.com \\ ${ }^{* *}$ National Institute of Technology, Dept. of Mathematics, Silchar, AS, India. \\ rupam.haloi15@gmail.com \\ ${ }^{* * *}$ Tripura University, Dept. of Mathematics, Agartala, TR, India. \\ binodtripathy@tripurauniv.in
}

Received: October 2018 | Accepted: November 2019

\section{Abstract:}

In this article, we introduce the concept of $\mu$-statistical convergence and $\mu$-density convergence of sequences of functions defined on a compact subset $D$ of the probabilistic normed space $(X, N, *)$, where $\mu$ is a finitely additive two valued measure. In particular, we introduce the notions of $\mu$-statistical uniform convergence as well as $\mu$-statistical point-wise convergence of sequences of functions in probabilistic normed space (in short PN-space) and we give some characterization results on these two convergences of sequences of functions in PN-space. We have also observed that $\mu$-statistical uniform convergence of sequences of functions in PN-spaces inherits the basic properties of uniform convergence.

Keywords: Probabilistic normed space; $\mu$-statistical convergence; $\mu$-density convergence; Point-wise and uniform convergence AP O condition.

MSC (2010): 40A30, 40G15, 54E70.

\section{Cite this article as (IEEE citation style):}

M. Sen, R. Haloi and B. Tripathy, " $\mu$-Statistically convergent function sequences in probabilistic normed linear spaces", Proyecciones (Antofagasta, On line), vol. 38, no. 5, pp. 1039 1056, Dec. 2019, doi: 10.22199/issn.0717-6279-2019-050066. [Accessed dd-mm-yyyy].

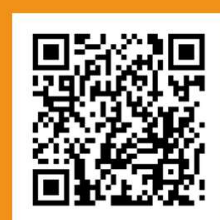

Article copyright: (c) 2019 Mausumi Sen, Rupan Haloi and Binod Chandra Tripathy. This is an open access article distributed under the terms of the Creative Commons Licence, which permits unrestricted use and distribution provided the original author and source are credited. 


\section{Introduction}

The notion of probabilistic normed space was first introduced by Šerstnev [21] in 1963. In this theory, it has been observed that these spaces are nothing but the generalization of real linear spaces in which the norm of a vector is a distribution function rather than just a number. Situations where crisp norm is unable to measure the length of a vector accurately, the notion of probabilistic norm happens to be useful. Its theory is important as a generalization of deterministic results of normed linear spaces and provides us important tools suitable for the study of topological spaces, geometry of nuclear physics, linear operators, continuity properties, convergence of random variables etc. Later this theory was generalised by many authors $[1,13]$. The idea of statistical convergence was first introduced by Steinhaus [22] as well as by Fast [11] in 1951. Later on, Connor [5] and Fridy [12] have shown that convergent sequences are statistically convergent, but the converse is not true in general. The theory of statistical convergence has been investigated by many authors in recent papers $[8,9,18]$. Karakus [15] has introduced the idea of statistical convergence in $P N$-space in 2007. The studies on sequence spaces in $P N$-space are due to $[23,24,25,26,28]$. The reader may refer to recent textbooks Başar [4] and Mursaleen [17] for sequence spaces, matrix transformations and related topics on summability theory.

In [6], Connor has given an extension to the notion of statistical convergence, where the asymptotic density is replaced by a finitely additive two valued measure $\mu$. From Connor [6] itself, it is known that if a sequence is $\mu$-density convergent with respect to a probabilistic norm $N$, then the sequence is $\mu$-statistically convergent with respect to the same probabilistic norm $N$; but the converse is not true in general. These two definitions are equivalent, if $\mu$ has the additive property for null sets (also called the APO condition), which states that, if given a collection $\left\{A_{i}\right\}_{i \in \mathbf{N}} \subseteq \Gamma$ of mutually disjoint $\mu$-null sets (i.e., $\mu\left(A_{i}\right)=0$, for all $i \in \mathbf{N}$ ) such that $A_{i} \cap A_{j}=\emptyset$, for $i \neq j$, then there exists a collection $\left\{B_{i}\right\}_{i \in \mathbf{N}} \subseteq \Gamma$ with $\left|A_{i} \triangle B_{i}\right|<\infty$, for each $i \in \mathbf{N}$ and $B=\cup_{i} B_{i} \in \Gamma$ with $\mu(B)=0$.

The notion of convergence of sequence of function is also considered in measure theory. Wilczyński [29] studied the statistical convergence of sequences of functions in 2000. Some classification may also be found in [16].

This paper is a continuation of [10], where it is shown that $\mu$-statistical convergence and convergence in $\mu$-density are equivalent only if $\mu$ has the 
APO condition. Further, it is also observed that function sequences preserve $\mu$-statistical convergence on a subset $D$ of $\mathbf{R}$, also called $\mu$-statistically conservative on $D$. In this article, we show that this real space can be replaced by a probabilistic normed space $(X, N, *)$. In other sense, our main objective of the present paper is to introduce and investigate the convergence in $\mu$-density and $\mu$-statistical convergence of sequences of functions in $P N$-spaces. We also introduce the concepts of $\mu$-statistical uniform convergence and $\mu$-statistical point-wise convergence for the sequences of functions in $P N$-space. We show that all the results and characterizations for the sequences of functions in real space shown in [10], can be extended to the $v$-space $(X, N, *)$. Moreover, we observe that $\mu$-statistical uniform convergence for the sequences of functions in $P N$-space inherits the basic properties of uniform convergence.

A brief sketch of the paper is as follows : Section 2 gives some basic definitions and examples related to the topic. We give the definitions of $\mu$-statistical convergence and convergence in $\mu$-density for the sequences of functions in $P N$-spaces and discussed some of their properties and characterizations in section 3 . Section 4 deals with the function sequences that preserves the $\mu$-statistical convergence in $P N$-space.

\section{Preliminaries}

Throughout the paper, $\mathbf{N}, \mathbf{R}$ and $\mathbf{R}^{+}$denote the sets of natural, real and non-negative real numbers, respectively. Moreover, $\mu$ will denote a complete $\{0,1\}$-valued finitely additive measure defined on a field $\Gamma$ of subsets of $\mathbf{N}$ that contains all finite subsets of $\mathbf{N}$ and suppose that $\mu(A)=0$, if $|A|<\infty$; if $A \subset B$ and $\mu(B)=0$, then $\mu(A)=0$; and $\mu(\mathbf{N})=1$.

Definition 2.1. A function $f: \mathbf{R}^{+} \rightarrow[0,1]$ is called a distribution function if it is non-decreasing, left-continuous with $\inf _{t \in \mathbf{R}} f(t)=0$ and $\sup _{t \in \mathbf{R}} f(t)=$ 1 .

Let $\mathcal{D}$ denotes the set of all distribution functions.

Definition 2.2. [19] A binary operation $*:[0,1] \times[0,1] \rightarrow[0,1]$ is said to be a continuous t-norm if it satisfies the following conditions, for all $a, b, c, d \in[0,1]$ :

1. $a * 1=a$,

2. $a * b=b * a$, 
3. $a * b \leq c * d$, whenever $a \leq c$ and $b \leq d$,

4. $(a * b) * c=a *(b * c)$.

Definition 2.3. [1] A triplet $(X, N, *)$ is called a probabilistic normed space (in short a $P N$-space) if $X$ is a real vector space, $N$ a mapping from $X$ into $\mathcal{D}$ (for $x \in X$, the distribution function $N(x)$ is denoted by $N_{x}$ and $N_{x}(t)$ is the value of $N_{x}$ at $t \in \mathbf{R}$ ) and $*$ a t-norm satisfying the following conditions:

1. $N_{x}(0)=0$,

2. $N_{x}(t)=1$, for all $t>0$ if and only if $x=0$,

3. $N_{\alpha x}(t)=N_{x}\left(\frac{t}{|\alpha|}\right)$, for all $\alpha \in \mathbf{R} \backslash\{0\}$,

4. $N_{x+y}(s+t) \geq N_{x}(s) * N_{y}(t)$, for all $x, y \in X$ and $s, t \in \mathbf{R}^{+}$.

Example 2.4. [2] Let $(X,\|\|$.$) be a normed linear space. Let a * b=$ $\min \{a, b\}$, for all $a, b \in[0,1]$ and $N_{x}(t)=\frac{t}{t+\|x\|}, x \in X$ and $t \geq 0$. Then $(X, N, *)$ is a $P N$-space.

Definition 2.5. [2] A sequence $x=\left(x_{k}\right)$ in a $P N$-space $(X, N, *)$ is said to be convergent to $L \in X$ with respect to the probabilistic norm $N$, if for every $\varepsilon>0$ and $\lambda \in(0,1)$, there exists a positive integer $k_{0}$ such that $N_{x_{k}-L}(\varepsilon)>1-\lambda$, whenever $k \geq k_{0}$. In this case, we write $N-\lim x=L$.

Definition 2.6. [2] A sequence $x=\left(x_{k}\right)$ in a $P N$-space $(X, N, *)$ is said to be Cauchy sequence, if for every $\varepsilon>0$ and $\lambda \in(0,1)$, there exists a positive integer $k_{0}$ such that $N_{x_{k}-x_{m}}(\varepsilon)>1-\lambda$, for all $k, m \geq k_{0}$.

Definition 2.7. [2] A sequence $x=\left(x_{k}\right)$ in a $P N$-space $(X, N, *)$ is said to be bounded, if there exists $\varepsilon>0$ such that $N_{x_{k}}(\varepsilon)>1-\lambda$, for every $\lambda \in(0,1)$ and for all $k$.

Definition 2.8. [6] A sequence $x=\left(x_{k}\right)$ is said to be $\mu$-density convergent to $L \in X$ with respect to the probabilistic norm $N$, if there is an $A \in \Gamma$ with $\mu(A)=1$ such that $\left(x_{k}-L\right)_{k \in A}$ is convergent to 0 with respect to the probabilistic norm $N$. 
Definition 2.9. [6] A sequence $x=\left(x_{k}\right)$ is said to be $\mu$-statistically convergent to $L$ with respect to the probabilistic norm $N$, if for every $\varepsilon>0$ and $\lambda \in(0,1), \mu\left(\left\{k \in \mathbf{N}: N_{x_{k}-L}(\varepsilon) \leq 1-\lambda\right\}\right)=0$. We denote it by $\mu-\operatorname{stat}_{N}-\lim x=L$.

Definition 2.10. [14] Let $D$ be a subset of a $P N$-space $(X, N$,*), then $D$ is said to be compact if each sequence of elements of $X$ has a convergent subsequence in $D$.

\section{3. $\mu$-statistically and $\mu$-density convergent function sequences in PN-space}

In this section, we introduce the following definitions on $\mu$-density and $\mu$-statistically convergent function sequences in $P N$-space. Let $D$ be a compact subset of $(X, N, *)$ and let $\left(f_{n}\right)$ be a sequence of functions on $D$.

Definition 3.1. The function sequence $\left(f_{n}\right) \mu$-density point-wise converges to $f$ with respect to probabilistic norm $N$ on $D$ if and only if for each $t \in D$ and for all $\varepsilon>0, \lambda \in(0,1)$, there exists $A_{t} \in \Gamma$, with $\mu\left(A_{t}\right)=1$ and a number $n_{0}=n_{0}(\varepsilon, \lambda, t) \in A_{t}$ such that $N_{f_{n}(t)-f(t)}(\varepsilon)>1-\lambda$, for all $n \geq n_{0}$ and $n \in A_{t}$.

In this case, we write $f_{n} N f(\mu-$ density) on $D$.

Definition 3.2. The function sequence $\left(f_{n}\right) \mu$-density uniformly converges to $f$ with respect to probabilistic norm $N$ on $D$ if and only if for all $\varepsilon>0$ and $\lambda \in(0,1)$, there exists $A \in \Gamma$, with $\mu(A)=1$ and a number $n_{0}=$ $n_{0}(\varepsilon, \lambda) \in A$ such that $N_{f_{n}(t)-f(t)}(\varepsilon)>1-\lambda$, for all $n \geq n_{0}, n \in A$ and for every $t \in D$.

In this case, we write $f_{n} N f(\mu-$ density) on $D$.

Definition 3.3. The function sequence $\left(f_{n}\right) \mu$-statistically point-wise converges to $f$ with respect to probabilistic norm $N$ on $D$ if and only if for each $t \in D$ and for all $\varepsilon>0, \lambda \in(0,1)$, we have $\mu\left(\left\{n \in \mathbf{N}: N_{f_{n}(t)-f(t)}(\varepsilon) \leq\right.\right.$ $1-\lambda\})=0$.

In this case, we write $f_{n} N f(\mu-s t a t)$ on $D$.

Definition 3.4. The sequence $\left(f_{n}\right)$ of bounded functions on $D$ is said to be $\mu$-statistically uniformly convergent to $f$ with respect to probabilistic norm $N$ if and only if for all $\varepsilon>0$ and $\lambda \in(0,1)$, we have $\mu(\{n \in \mathbf{N}$ : $\left.\left.N_{f_{n}(t)-f(t)}(\varepsilon) \leq 1-\lambda\right\}\right)=0$, for every $t \in D$. 
In this case, we write $f_{n} N f(\mu-$ stat) on $D$.

As in the ordinary case, if a function sequence is $\mu$-density point-wise convergent with respect to a probabilistic norm $N$, then the function sequence is $\mu$-statistically point-wise convergent with respect to the probabilistic norm $N$; and, of course for bounded functions, if a function sequence is $\mu$-density uniformly convergent with respect to a probabilistic norm $N$, then the function sequence is $\mu$-statistically uniformly convergent with respect to the probabilistic norm $N$. However, if $\mu$ has the APO condition, then $\mu$-density point-wise convergence with respect to a probabilistic norm $N$ and $\mu$-statistically point-wise convergence with respect to the probabilistic norm $N$ are equivalent; and $\mu$-density uniform convergence with respect to a probabilistic norm $N$ and $\mu$-statistically uniformly convergence with respect to the probabilistic norm $N$ are equivalent.

The following result is a $\mu$-statistical analogue of a well known result in the sense of a probabilistic norm $N$.

Theorem 3.5. Let $\left(f_{n}\right)$ be a sequence of continuous functions on $D$. If $f_{n} N f$ ( $\mu$-density) on $D$, then $f$ is continuous with respect to the probabilistic norm $N$ on $D$.

Proof. Suppose that $f_{n} N f(\mu-$ density $)$ on $D$. Then, for every $\varepsilon>0$ and $\gamma \in(0,1)$, there exists $A \in \Gamma$, with $\mu(A)=1$ and $n_{0}=n_{0}(\varepsilon, \lambda)$ in $A$ such that

$$
N_{f_{n}(t)-f(t)}(\varepsilon / 3)>1-\gamma,
$$

for all $n \geq n_{0}, n \in A$ and for all $t \in D$.

Let $t_{0} \in D$. Since $f_{n_{0}}$ is continuous at $t_{0} \in D$, so for all $\varepsilon>0$ and $\gamma \in(0,1)$

$$
N_{f_{n_{0}}(t)-f_{n_{0}}\left(t_{0}\right)}(\varepsilon / 3)>1-\gamma .
$$

Choose $\lambda \in(0,1)$ such that $(1-\gamma) *(1-\gamma) *(1-\gamma)>1-\lambda$. Then, for all $t \in D$, we have

$$
\begin{aligned}
& \mathrm{N}_{f(t)-f\left(t_{0}\right)}(\varepsilon)=N_{\left\{f(t)-f_{n_{0}}(t)+f_{n_{0}}(t)-f_{n_{0}}\left(t_{0}\right)\right\}+\left\{f_{n_{0}}\left(t_{0}\right)-f\left(t_{0}\right)\right\}}(2 \varepsilon / 3+\varepsilon / 3) \\
\geq & N_{\left\{f(t)-f_{n_{0}}(t)\right\}+\left\{f_{n_{0}}(t)-f_{n_{0}}\left(t_{0}\right)\right\}}(2 \varepsilon / 3) * N_{f_{n_{0}}\left(t_{0}\right)-f\left(t_{0}\right)}(\varepsilon / 3) \\
\geq & N_{f(t)-f_{n_{0}}(t)}(\varepsilon / 3) * N_{f_{n_{0}}(t)-f_{n_{0}}\left(t_{0}\right)}(\varepsilon / 3) * N_{f_{n_{0}}\left(t_{0}\right)-f\left(t_{0}\right)}(\varepsilon / 3) \\
> & (1-\gamma) *(1-\gamma) *(1-\gamma) \\
> & 1-\lambda .
\end{aligned}
$$

Since $t_{0} \in D$ is arbitrary, so $f$ is continuous with respect to the probabilistic norm $N$ on $D$. 
Corollary 3.6. Let $\left(f_{n}\right)$ be a sequence of continuous functions on a compact subset $D$ of $(X, N, *)$ and let $\mu$ be a measure with the APO condition. If $f_{n} N f(\mu-$ stat $)$ on $D$, then $f$ is continuous with respect to the probabilistic norm $N$ on $D$.

Example 3.7. Let $\mathcal{D}$ be a compact subset of $(X, N, *)$ and $\mu(A)=1$.

Define a function $f_{n}: D \rightarrow X$ such that

$$
f_{n}(t)= \begin{cases}1, & \text { if } n \notin A \\ \frac{3 n t}{1+n^{3} t^{3}}, & \text { if } n \in A\end{cases}
$$

for all $t \in D$.

Then, we have $f_{n} N f$ ( $\mu$-density) on $D$ and consequently, we have $f_{n} N f$ ( $\mu-$ stat) on $D$. Though $f_{n}$ and $f$ are all continuous on the compact subset $D$ of the $P N$-space $(X, N, *)$, it follows from Definition 3.4 that $\left(f_{n}\right)$ is not $\mu$-statistically uniformly convergent, as

$$
\mu\left(\left\{n \in \mathbf{N}: N_{f_{n}(t)-f(t)}(\varepsilon) \leq 1-\lambda\right\}\right)=1 .
$$

The next result is an analogue of Dini's theorem in probabilistic normed space $(X, N, *)$.

Theorem 3.8. Let $\mu$ be a measure with the APO condition. Let $D$ be a compact subset of a $P N$-space $(X, N, *)$ and let $\left(f_{n}\right)$ be a function sequence that are continuous with respect to the probabilistic norm $N$ on $D$. Suppose that $f$ is continuous with respect to the probabilistic norm $N$ and $f_{n} N f(\mu-s t a t)$ on $D$. Furthermore, let $\left(f_{n}\right)$ be monotonic decreasing on $D$, i.e., $f_{n}(t) \geq f_{n+1}(t)$, for every $t \in D$ and $n=1,2, \ldots$ Then $f_{n} N f(\mu-s t a t)$ on $D$.

Proof. Let $g_{n}(t)=f_{n}(t)-f(t)$. Then, by the hypothesis, each $g_{n}$ is continuous and $g_{n} N 0(\mu-$ stat $)$ on $D$. Moreover, $\left(g_{n}\right)$ is a monotonic decreasing sequence on $D$. Since $g_{n} N 0(\mu-$ stat $)$ on $D$ and $\mu$ has the APO condition, so $g_{n} N 0(\mu-$ density) on $D$. Thus, for every $\varepsilon>0, \gamma \in(0,1)$ and for each $t \in D$, there exists $A_{t} \in \Gamma$, with $\mu\left(A_{t}\right)=1$ and $n(t)=$ $n(\varepsilon, \lambda, t) \in A_{t}$ such that $N_{g_{n}(t)}(\varepsilon / 2)>1-\gamma$, for all $n \geq n(t)$, and $n \in A_{t}$.

Since $g_{n(t)}$ is continuous at $t \in D$ with respect to the probabilistic norm $N$, so for every $\varepsilon>0$ and $\gamma \in(0,1)$, there exists an open set $K(t)$ containing $t$ such that

$$
N_{g_{n(t)}(x)-g_{n(t)}(t)}(\varepsilon / 2)>1-\gamma
$$


for all $x \in K(t)$. Now, we choose $\lambda \in(0,1)$ such that $(1-\gamma) *(1-\gamma)>1-\lambda$. Then, by monotonicity, we have for $\varepsilon>0$ and $\gamma \in(0,1)$

$$
\mathrm{N}_{g_{n}(x)}(\varepsilon) \geq N_{g_{n(t)}(x)}(\varepsilon)
$$

$=N_{g_{n(t)}(x)-g_{n(t)}(t)+g_{n(t)}(t)}(\varepsilon / 2+\varepsilon / 2)$

$\geq N_{g_{n(t)}(x)-g_{n(t)}(t)}(\varepsilon / 2) * N_{g_{n(t)}(t)}(\varepsilon / 2)$

$>(1-\gamma) *(1-\gamma)$

$>1-\lambda$,

for all $n \geq n(t), n \in A_{t}$ and for every $x \in K(t)$.

Since $D$ is a compact set and $D \subset \bigcup_{t \in D} K(t)$, so by the Heine-Borel theorem, $D$ has a finite open covering such that $D \subset K\left(t_{1}\right) \cup K\left(t_{2}\right) \cup \ldots \cup$ $K\left(t_{m}\right)$. Let $A:=A_{t_{1}} \cap A_{t_{2}} \cap \ldots \cap A_{t_{m}}$ and $N=\max \left\{n\left(t_{1}\right), \ldots, n\left(t_{m}\right)\right\}$. We observe that $\mu(A)=1$. Then, for every $\varepsilon>0$ and $\lambda \in(0,1)$, we have $N_{g_{n}(x)}(\varepsilon)>1-\lambda$, for all $x \in D, n \geq N$ and $n \in A$. Hence, $g_{n}{ }^{N} 0(\mu-$ density) on $D$. Consequently, $g_{n}{ }^{N}(\mu-$ stat) on $D$, and hence the proof.

We, now give the following two definitions, the first one on $\mu$-Cauchy function sequence and the second one on $\mu$-statistically uniformly Cauchy, both in the sense of probabilistic norm $N$, and then give the Cauchy criterion for $\mu$-statistically uniformly convergence in $\mathrm{PN}$-space.

Definition 3.9. Let $D$ be a compact subset of a $P N$-space $(X, N, *)$. Let $\left(f_{n}\right)$ be a sequence of functions on $D$. Then $\left\{f_{n}(t)\right\}$ is said to be $\mu$-Cauchy with respect to the probabilistic norm $N$, if for every $\varepsilon>0$ and $\lambda \in(0,1)$, there exists a $M=M(\varepsilon, \lambda, t) \in \mathbf{N}$ such that

$$
\mu\left(\left\{n: N_{f_{n}(t)-f_{M}(t)}(\varepsilon)>1-\lambda\right\}\right)=1 .
$$

Definition 3.10. The sequence $\left(f_{n}\right)$ of bounded functions on the compact subset $D$ of the $P N$-space $(X, N, *)$ is said to be $\mu$-statistically uniformly Cauchy on $D$ with respect to probabilistic norm $N$, if for every $\varepsilon>0$ and $\lambda \in(0,1)$, there exists a $M=M(\varepsilon, \lambda) \in \mathbf{N}$ such that

$$
\mu\left(\left\{n: N_{f_{n}(t)-f_{M}(t)}(\varepsilon)>1-\lambda\right\}\right)=1 .
$$

To prove the Cauchy criterion for $\mu$-statistically uniformly convergence in $P N$-space, we use the following lemma.

Lemma 3.11. Let $D$ be a compact subset of a $P N$-space $(X, N, *)$. Let $\left(f_{n}\right)$ be a sequence of functions on $D$. Then $\left\{f_{n}(t)\right\}$ is $\mu$-statistically convergent with respect to the probabilistic norm $N$ if and only if $\left\{f_{n}(t)\right\}$ is $\mu$-Cauchy with respect to the probabilistic norm $N$. 
Proof. Assume that $\left\{f_{n}(t)\right\}$ is $\mu$-statistically convergent to $f(t)$ with respect to the probabilistic norm $N$. Then, for every $\varepsilon>0$ and $\lambda \in(0,1)$, we have

$$
\mu\left(\left\{n: N_{f_{n}(t)-f(t)}(\varepsilon / 2)>1-\lambda\right\}\right)=1 .
$$

Now, we select an $M=M(\varepsilon, \lambda, t) \in \mathbf{N}$ such that $N_{f_{M}(t)-f(t)}(\varepsilon / 2)>1-\lambda$. Then, by choosing $\gamma \in(0,1)$ such that $(1-\lambda) *(1-\lambda)>(1-\gamma)$, we have

$$
\mathrm{N}_{f_{n}(t)-f_{M}(t)}(\varepsilon)=N_{f_{n}(t)-f(t)+f(t)-f_{M}(t)}(\varepsilon / 2+\varepsilon / 2)
$$

$\geq N_{f_{n}(t)-f(t)}(\varepsilon / 2) * N_{f_{M}(t)-f(t)}(\varepsilon / 2)$

$>(1-\lambda) *(1-\lambda)$

$>1-\gamma$

which yields that $\mu\left(\left\{n: N_{f_{n}(t)-f_{M}(t)}(\varepsilon)>1-\gamma\right\}\right)=1$. Since $\varepsilon$ and $\gamma$ are arbitrary, hence $\left\{f_{n}(t)\right\}$ is $\mu$-Cauchy.

Conversely, assume that $\left\{f_{n}(t)\right\}$ is $\mu$-Cauchy with respect to the probabilistic norm $N$. Then, for every $\varepsilon>0$ and $\lambda \in(0,1)$, there exists a $M=M(\varepsilon, \lambda, t) \in \mathbf{N}$ such that

$$
\mu\left(\left\{n: N_{f_{n}(t)-f_{M}(t)}(\varepsilon)>1-\lambda\right\}\right)=1 .
$$

We claim that $\left\{f_{n}(t)\right\}$ is $\mu$-statistically convergent to $f(t)$ with respect to the probabilistic norm $N$. Let $\varepsilon>0$ and $\gamma \in(0,1)$ be given. If possible, suppose that

$$
\mu\left(\left\{n: N_{f_{n}(t)-f(t)}(\varepsilon / 2)>1-\gamma\right\}\right)=0 .
$$

Now, we can select an $M=M(\varepsilon, \lambda, t) \in \mathbf{N}$ such that

$$
N_{f_{M}(t)-f(t)}(\varepsilon / 2)>1-\gamma .
$$

We choose $\lambda \in(0,1)$ such that $(1-\gamma) *(1-\gamma)>(1-\lambda)$. Then, we have

$$
\mu\left(\left\{n: N_{f_{n}(t)-f_{M}(t)}(\varepsilon)>1-\lambda\right\}\right)=0,
$$

which contradicts our hypothesis. Thus, we have

$$
\mu\left(\left\{n: N_{f_{n}(t)-f(t)}(\varepsilon) \leq 1-\gamma\right\}\right)=0,
$$

consequently $\left\{f_{n}(t)\right\}$ is $\mu$-statistically convergent to $f(t)$ with respect to the probabilistic norm $N$.

Now, we are ready to give the Cauchy criterion for $\mu$-statistically uniformly convergence in $\mathrm{PN}$-space.

Theorem 3.12. Let $\mu$ be a measure with the APO condition, and $\left(f_{n}\right)$ be a sequence of bounded functions on a compact subset $D$ of the $P N$-space $(X, N, *)$. Then $\left(f_{n}\right)$ is $\mu$-statistically uniformly convergent with respect to the probabilistic norm $N$ on $D$ if and only if $\left(f_{n}\right)$ is $\mu$-statistically uniformly Cauchy with respect to the probabilistic norm $N$ on $D$. 
Proof. Suppose that $\left(f_{n}\right)$ converges $\mu$-statistically uniformly to a function $f$ with respect to probabilistic norm $N$ on $D$. Then, for every $\varepsilon>0$ and $\gamma \in(0,1)$, we have

$$
\mu\left(\left\{n: N_{f_{n}(t)-f(t)}(\varepsilon / 2)>1-\gamma\right\}\right)=1,
$$

for every $t \in D$. We select an $M=M(\varepsilon, \lambda) \in \mathbf{N}$ in such a way that

$$
N_{f_{M}(t)-f(t)}(\varepsilon / 2)>1-\gamma .
$$

Choosing $\lambda \in(0,1)$ such that $(1-\gamma) *(1-\gamma)>(1-\lambda)$, we have

$$
\mu\left(\left\{n: N_{f_{n}(t)-f_{M}(t)}(\varepsilon)>1-\lambda\right\}\right)=1 .
$$

Since $\varepsilon$ is arbitrary, so $\left(f_{n}\right)$ is $\mu$-statistically uniformly Cauchy with respect to probabilistic norm $N$ on $D$.

Conversely, suppose that $\left(f_{n}\right)$ is $\mu$-statistically uniformly Cauchy with respect to the probabilistic norm $N$ on $D$. Let $t \in D$ fixed. We have, from (3.1) that for every $\varepsilon>0$ and $\lambda \in(0,1)$, there exists a $M=M(\varepsilon, \lambda) \in \mathbf{N}$ such that

$$
\mu\left(\left\{n: N_{f_{n}(t)-f_{M}(t)}(\varepsilon)>1-\lambda\right\}\right)=1 .
$$

Thus, $\left\{f_{n}(t)\right\}$ is $\mu$-Cauchy, and so by Lemma 3.11, $\left\{f_{n}(t)\right\}$ converges $\mu$ statistically to $f(t)$. Thus, $f_{n} N f(\mu-s t a t)$ on $D$.

Now, we show that this convergence is uniform. Since $\mu$ has the APO condition, so by the relation (3.1), there exists a $A \in \Gamma$ with $\mu(A)=1$ such that

$$
N_{f_{n}(t)-f_{M}(t)}(\varepsilon / 2)>1-\lambda,
$$

for all $n \geq M, n \in A$ and $\lambda \in(0,1)$. So, for every $\varepsilon>0$ and $\lambda \in(0,1)$, there exists $A \in \Gamma$ with $\mu(A)=1$ and $M=M(\varepsilon, \lambda) \in \mathbf{N}$ such that

$$
N_{f_{n}(t)-f_{m}(t)}(\varepsilon)>1-\lambda,
$$

for all $n, m \geq M, n, m \in A$ and for every $t \in D$.

In (3.2), for fixed $n$ and $m \rightarrow \infty$, we have, for every $\varepsilon>0$ and $\lambda \in(0,1)$, there exists $A \in \Gamma$ with $\mu(A)=1$ and an $M=M(\varepsilon, \lambda) \in \mathbf{N}$ such that

$$
N_{f_{n}(t)-f(t)}(\varepsilon)>1-\lambda
$$

for all $n \geq M, n \in A$ and for every $t \in D$. Hence $f_{n} N f$ ( $\mu-$ density) on $D$. Consequently, $f_{n} N f(\mu-s t a t)$ on $D$. 


\section{4. $\mu$-statistically conservative function sequences in $P N$-space}

In 1999, Kolk [16] studied function sequences $\left(f_{n}\right)$ that preserve convergence (or conservative) on $D \subset \mathbf{R}$. This definition of function sequence preserving convergence was extended to the function sequence preserving $\mu$-statistical convergence by Duman and Orhan [10] in 2004. In this section, analogously, we describe the function sequences which preserves the $\mu$-statistical convergence in $P N$-space. A sequential characterization of the continuity of $\mu$-statistical limit functions of $\mu$-statistically uniformly convergent function sequences has also been given.

First we introduce the following definition in the sense of probabilistic norm $N$.

Definition 4.1. Let $D \subset(X, N, *)$ and let $\left(f_{n}\right)$ be a sequence of functions on $D$. Then $\left(f_{n}\right)$ is called a function sequence preserving $\mu$-statistical convergence with respect to a probabilistic norm $N$ (or $\mu$-statistically conservative in $P N$-space) on $D$ if the transformed sequence $\left\{f_{n}(t)\right\}$ converges $\mu$-statistically with respect to the probabilistic norm $N$, for each $\mu$-statistically convergent sequence $t=\left(t_{n}\right)$ with respect to the probabilistic norm $N$ from $D$. If $\left(f_{n}\right)$ is $\mu$-statistically conservative in $P N$-space and preserves the limits of all $\mu$-statistically convergent sequences with respect to a probabilistic norm $N$ from $D$, then $\left(f_{n}\right)$ is called $\mu$-statistically regular with respect to the probabilistic norm $N$ on $D$.

Hence, if $\left(f_{n}\right)$ is conservative with respect to probabilistic norm $N$ on $D$, then $\left(f_{n}\right)$ is $\mu$-statistically conservative with respect to probabilistic norm $N$ on $D$, but the converse of this result is not true, which is shown by the following example.

Example 4.2. Let $A \in \Gamma$ such that $\mathbf{N} \backslash A$ is infinite and $\mu(A)=1$. Then, we define $f_{n}: D \rightarrow X$ by

$$
f_{n}(t)= \begin{cases}0, & n \in A \\ 1, & n \notin A\end{cases}
$$

for all $t \in D$. Assume that $\left(t_{n}\right)$ is an arbitrary sequence from $D$ such that $\mu-\operatorname{stat}_{N}-\lim t_{n}=L$. Then, for every $\varepsilon>0$ and $\lambda \in(0,1)$, we have

$$
\mu\left(\left\{n: N_{f_{n}(t)}(\varepsilon) \leq 1-\lambda\right\}\right)=\mu(\mathbf{N} \backslash A)=0 .
$$

Hence, $\mu-$ stat $_{N}-\lim f_{n}\left(t_{n}\right)=0$, so $\left(f_{n}\right)$ is $\mu$-statistically conservative with respect to probabilistic norm $N$ on $D$. But we observe that $\left(f_{n}\right)$ is not conservative with respect to probabilistic norm $N$ on $D$. 
In order to prove our next theorem, we use the following lemma. The proof of the lemma is easy, so omitted.

Lemma 4.3. Let $\mu$ be a measure with the APO condition. If $\left(f^{r}\right)$ is a countable collection of function sequences that are convergent in $\mu$-density with respect to the probabilistic norm $N$, then there exists $\lambda: \mathbf{N} \rightarrow \mathbf{N}$ such that $N-\lim _{k} f_{\lambda(k)}^{r}$ exists, for each fixed $r$ and $\mu(\{\lambda(k): k \in \mathbf{N}\})=1$.

Theorem 4.4. Let $\mu$ be a measure with the APO condition and let the function sequence $\left(f_{n}\right)$ be defined on a compact set $D$ of the $P N$-space $(X, N, *)$. Then $\left(f_{n}\right)$ preserves $\mu$-statistical convergence with respect to a probabilistic norm $N$ on $D$ if and only if $\left(f_{n}\right)$ is $\mu$-statistically uniformly convergent with respect to the probabilistic norm $N$ on $D$ and converges to a continuous function.

Proof. First, we suppose that $\left(f_{n}\right)$ preserves $\mu$-statistical convergence with respect to probabilistic norm $N$ on $D$. Now, for each $x \in D$, we choose the sequence $\left(u_{n}\right)=(x, x, \ldots)$. Since $\mu-\operatorname{stat}_{N}-\lim u_{n}=x$, so $\mu-\operatorname{stat}_{N}-$ $\lim f_{n}\left(u_{n}\right)$ exists, hence, for all $x \in D$, we have $\mu-\operatorname{stat}_{N}-\lim f_{n}(x)=f(x)$. We claim that, $f$ is continuous on $D$ with respect to the probabilistic norm $N$. By the method of contradiction, we assume that $f$ is not continuous at a point $x_{0} \in D$. Then, there exists a sequence $\left(v_{n}\right)$ in $D$ such that $N-\lim v_{n}=x_{0}$, but $N-\lim f\left(v_{n}\right) \neq f\left(x_{0}\right)$. Since $\left(f_{n}\right)$ is $\mu$-statistically point-wise convergent to $f$ with respect to probabilistic norm $N$ on $D$ and $\mu$ has the APO condition, so we have $f_{n} N f(\mu-d e n s i t y)$ on $D$. Hence, for each $i$, we have $\left\{f_{n}\left(v_{i}\right)-f\left(v_{i}\right)\right\} N 0$ ( $\mu$-density). Thus, it follows from Lemma 4.3, that there exists $\lambda: \mathbf{N} \rightarrow \mathbf{N}$ with $\mu(\{\lambda(n): n \in \mathbf{N}\})=1$ such that

$$
N-\lim _{n}\left[f_{\lambda(n)}\left(v_{i}\right)-f\left(v_{i}\right)\right]=0
$$

for each $i$. Now, we can choose, by the diagonal process ([3], p. 192), an index sequence $\left(k_{n}\right)$ such that $\mu\left(\left\{k_{n}: n \in \mathbf{N}\right\}\right)=1$ and

$$
N-\lim _{n}\left[f_{k_{n}}\left(v_{n}\right)-f\left(v_{n}\right)\right]=0 .
$$

Now, we define $\left(x_{j}\right)$ by

$$
x_{j}=\left\{\begin{array}{cc}
x_{0}, \quad j=k_{n} \text { and } j \text { is odd } \\
v_{n}, \quad j=k_{n} \text { and } j \text { is even } \\
0, & \text { otherwise }
\end{array}\right.
$$


Hence, $x_{j} N x_{0}(\mu-$ density $)$ and so we have $\mu-$ stat $_{N}-\lim x_{j}=x_{0}$. But, if $j=k_{n}$ and $j$ is odd, then $N-\lim f_{k_{n}}\left(x_{0}\right)=f\left(x_{0}\right)$ and if $j=k_{n}$ and $j$ is even, then

$$
N-\lim f_{k_{n}}\left(v_{n}\right)=N-\lim \left[f_{k_{n}}\left(v_{n}\right)-f\left(v_{n}\right)\right]+N-\lim f\left(v_{n}\right) \neq f\left(x_{0}\right) .
$$

Hence, $\left\{f_{j}\left(x_{j}\right)\right\}$ is not convergent in $\mu$-density with respect to probabilistic norm $N$, as the sequence $\left\{f_{j}\left(x_{j}\right)\right\}$ has two distinct sub-sequences of positive measure which converge to two different limit points. So, the sequence $\left\{f_{j}\left(x_{j}\right)\right\}$ doesn't converge $\mu$-statistically with respect to probabilistic norm $N$, which contradicts the hypothesis. Hence, $f$ must be continuous on $D$. Now, it remains to prove that $\left(f_{n}\right)$ is $\mu$-statistically uniformly convergent to $f$ with respect to probabilistic norm $N$ on $D$. Suppose that $\left(f_{n}\right)$ does not $\mu$-statistically uniformly converge to $f$ with respect to probabilistic norm $N$ on $D$, which implies that $\left(f_{n}\right)$ is not $\mu$-density uniformly convergent to $f$ with respect to probabilistic norm $N$ on $D$. Thus, for an arbitrary index sequence $\left(k_{n}\right)$ with $\mu\left(\left\{k_{n}: n \in \mathbf{N}\right\}\right)=1$, there exists a number $\varepsilon>0$ and numbers $x_{n} \in D$ such that

$$
N_{f_{k_{n}}\left(x_{n}\right)-f\left(x_{n}\right)}(\varepsilon) \leq 1-\gamma,
$$

for $\gamma \in(0,1)$. The sequence $\left(x_{n}\right)$ contains a convergent sub-sequence $\left(x_{n_{j}}\right)$ in the $P N$-space $(X, N, *)$ such that $\mu-\operatorname{stat}_{N}-\lim x_{n_{j}}=\alpha$ (say). Continuity of $f$ implies $N-\lim f\left(x_{n_{j}}\right)=f(\alpha)$. So, there is an index $j_{0}$ such that

$$
N_{f\left(x_{n_{j}}\right)-f(\alpha)}(\varepsilon)>1-\lambda
$$

for $\lambda \in(0,1)$ and $j \geq j_{0}$. For the same $j$ 's, we must have

$$
N_{f_{k_{j}}\left(x_{n_{j}}\right)-f(\alpha)}(\varepsilon) \leq 1-\lambda .
$$

Otherwise, if we have

$$
N_{f_{k_{n_{j}}}\left(x_{n_{j}}\right)-f(\alpha)}(\varepsilon)>1-\lambda,
$$

then, by choosing $\gamma \in(0,1)$, such that $(1-\lambda) *(1-\lambda)>(1-\gamma)$, and by using the relation given by (4.3), we have

$$
\begin{aligned}
& \mathrm{N}_{f_{k_{n_{j}}}\left(x_{n_{j}}\right)-f\left(x_{n_{j}}\right)}(\varepsilon)=N_{f_{k_{n}}}\left(x_{n_{j}}\right)-f(\alpha)+f(\alpha)-f\left(x_{n_{j}}\right) \\
\geq & N_{f_{k_{n_{j}}}}\left(x_{n_{j}}\right)-f(\alpha)(\varepsilon / 2) * N_{f\left(x_{n_{j}}\right)-f(\alpha)}(\varepsilon / 2) \\
> & (1-\lambda) *(1-\lambda) \\
> & 1-\gamma
\end{aligned}
$$


which is a contradiction to the fact given by (4.1). Now, we define the sequence $v=\left(v_{i}\right)$ by

$$
v_{i}=\left\{\begin{array}{cc}
\alpha, & i=k_{n_{j}} \text { and } i \text { is odd } \\
x_{n_{j}}, & i=k_{n_{j}} \text { and } i \text { is even, } \\
0, & \text { otherwise }
\end{array}\right.
$$

Then, we get $v_{i} N \alpha(\mu-$ density $)$. Hence $\mu-\operatorname{stat}_{N}-\lim v_{i}=\alpha$. But if $i=k_{n_{j}}$ and $i$ is odd, then $N-\lim f_{k_{n_{j}}}(\alpha)=f(\alpha)$ and if $i=k_{n_{j}}$ and $i$ is even, then we have, by $(4.2), N-\lim f_{k_{n_{j}}}\left(x_{n_{j}}\right) \neq f(\alpha)$. Thus, $\left\{f_{i}\left(x_{i}\right)\right\}$ is not convergent in $\mu$-density with respect to probabilistic norm $N$, the sequence $\left\{f_{i}\left(x_{i}\right)\right\}$ has two distinct sub-sequences of positive measure which converge to two distinct limit points. Consequently, the sequence $\left\{f_{i}\left(x_{i}\right)\right\}$ is not $\mu$-statistically convergent with respect to probabilistic norm $N$, which is a contradiction to the hypothesis. Hence, $\left(f_{n}\right)$ must be $\mu$-statistically uniformly convergent to $f$ with respect to probabilistic norm $N$ on $D$.

Conversely, suppose that $\left(f_{n}\right)$ converges $\mu$-statistically uniformly to $f$ with respect to the probabilistic norm $N$ on $D$ and $f$ is continuous. Let $t=\left(t_{n}\right)$ be a sequence which is $\mu$-statistically convergent with respect to probabilistic norm $N$ on $D$ with $\mu-s t_{N}-\lim t_{n}=t_{0}$. Since $\mu$ has the APO condition with $t_{n} N t_{0}(\mu-$ density), hence there is an index sequence $\left(n_{p}\right)$ such that

$$
N-\lim _{p} t_{n_{p}}=t_{0} \text { and } \mu\left(\left\{n_{p}: p \in \mathbf{N}\right\}\right)=1 .
$$

Continuity of $f$ at $t_{0}$ implies that

$$
N-\lim _{p} f\left(t_{n_{p}}\right)=f\left(t_{0}\right) .
$$

Hence, $f\left(t_{n}\right) N f\left(t_{0}\right)(\mu-$ density $)$. Then, for every $\varepsilon>0$ and $\gamma \in(0,1)$, there exists $A_{1} \in \Gamma$ with $\mu\left(A_{1}\right)=1$ and a number $n_{1} \in A_{1}$ such that

$$
N_{f\left(t_{n}\right)-f\left(t_{0}\right)}(\varepsilon / 2)>1-\gamma,
$$

for all $n \geq n_{1}$. Since $\mu$ has the APO condition, so the $\mu$-statistical uniform convergence with respect to probabilistic norm $N$ is equivalent to the $\mu$ density uniform convergence with respect to probabilistic norm $N$. Hence, for every $\varepsilon>0$ and $\gamma \in(0,1)$, there exists a $A_{2} \in \Gamma$ with $\mu\left(A_{2}\right)=1$ and a number $n_{2} \in A_{2}$ such that

$$
N_{f_{n}(x)-f(x)}(\varepsilon / 2)>1-\gamma
$$


for every $x \in D$ and for all $n \geq n_{2}$. Suppose that $N:=\max \left\{n_{1}, n_{2}\right\}$ and $A:=A_{1} \cap A_{2}$. Then, observe $\mu(A)=1$. We choose $\lambda \in(0,1)$ such that $(1-\gamma) *(1-\gamma)>1-\lambda$. Thus, by taking $x=t_{n}$, we have

$$
\mathrm{N}_{f_{n}\left(t_{n}\right)-f\left(t_{0}\right)}(\varepsilon)=N_{f_{n}\left(t_{n}\right)-f\left(t_{n}\right)+f\left(t_{n}\right)-f\left(t_{0}\right)}(\varepsilon)
$$

$\geq N_{f_{n}\left(t_{n}\right)-f\left(t_{n}\right)}(\varepsilon / 2) * N_{f\left(t_{n}\right)-f\left(t_{0}\right)}(\varepsilon / 2)$

$>(1-\gamma) *(1-\gamma)$

$>1-\lambda$

for all $n \geq N$ and $n \in A$. Hence, we have $f_{n}\left(t_{n}\right) N f\left(t_{0}\right)(\mu-$ density), and consequently, we have $\mu-s_{t a t}-\lim f_{n}\left(t_{n}\right)=f\left(t_{0}\right)$. Hence the proof.

Following is the necessary and sufficient condition for the continuity of $\mu$-statistical limit functions of function sequences in the $P N$-space $(X, N, *)$, which converge $\mu$-statistically uniformly on a compact subset $D$ of a $P N$ space $(X, N, *)$.

Theorem 4.5. Let $\mu$ be a measure with the APO condition and let the function sequence $\left(f_{n}\right)$ in the $v$-space $(X, N, *) \mu$-statistically uniform converges to a function $f$ with respect to the probabilistic norm $N$ on a compact subset $D$ of $(X, N, *)$. Then $\mu-$ stat $_{N}-\lim$ function $f$ is continuous on $D$ if and only if $\left(f_{n}\right)$ is $\mu$-statistically conservative on $D$.

Now, we give a characterization of the $\mu$-statistical regularity of sequences of functions in $P N$-space. If $\left(f_{n}\right)$ is $\mu$-statistically regular on $D \subset(X, N, *)$, then we have $\mu-\operatorname{stat}_{N}-\lim f_{n}(x)=x$, for all $x \in D$. Hence, by taking $f(x)=x$ in Theorem 4.4, we immediately get the following result.

Theorem 4.6. Let $\mu$ be a measure with the APO condition and let $\left(f_{n}\right)$ be a sequence of functions on a compact subset $D$ of the $P N$-space $(X, N, *)$. Then $\left(f_{n}\right)$ is $\mu$-statistically regular with respect to a probabilistic norm $N$ on $D$ if and only if $\left(f_{n}\right) \mu$-statistically uniform converges to the function $f$ with respect to the probabilistic norm $N$ on $D$, defined by $f(x)=x$, for all $x \in D$.

\section{Conclusion}

In this paper, we have introduced several notions like $\mu$-density pointwise convergence, $\mu$-density uniform convergence, $\mu$-statistical point-wise convergence, $\mu$-statistical uniform convergence, $\mu$-statistically conservative function sequences etc for the sequence of functions defined on a compact 
subset $D$ of a probabilistic normed space $(X, N, *)$ and proved several useful results for these notions. It has been observe that, if $\mu$ is a measure with the APO condition and $\left(f_{n}\right)$ is a sequence of functions defined on a compact set $D \subset(X, N, *)$, then $\left(f_{n}\right)$ preserves $\mu$-statistical convergence with respect to the probabilistic norm $N$ on $D$ if and only if $\left(f_{n}\right)$ is $\mu$ statistically uniformly convergent with respect to the probabilistic norm $N$ on $D$ and converges to a continuous function. Since every classical norm can induce a probabilistic norm, the results obtained here are more general than the corresponding normed space results.

\section{Acknowledgement}

This work has been supported by the Research Project SB/S4/MS:887/14 of SERB-Department of Science and Technology, Govt. of India.

The authors thank the referee for the comments on the first drat of the article those improved the presentation of the article.

\section{References}

[1] C. Alsina, B. Schweizer, and A. Sklar, "On the definition of a probabilistic normed space", Aequationes mathematicae, vol. 46, no. 1-2, pp. 91-98, Aug. 1993, doi: 10.1007/BF01834000.

[2] A. Asadollah and K. Nourouzi, "Convex sets in probabilistic normed spaces", Chaos, solutions \& fractals, vol. 36, no. 2, pp. 322-328, Apr. 2008, doi: 10.1016/j.chaos.2006.06.051.

[3] R. Bartle, Elements of real analysis, New York, NY: John Wiley and Sons Inc., 1964.

[4] F. Bassar, Summability theory and its applications, BenthameBooks, 2012, doi: $10.2174 / 97816080545231120101$.

[5] J. Connor, "The statistical and strong p-Cesàro convergence of sequences", Analysis, vol. 8, no. 1-2, pp. 47-63, 1988, doi: 10.1524/anly.1988.8.12.47.

[6] J. Connor, "Two valued measure and summability", Analysis, vol. 10, no. 4, pp. 373-385, 1990, doi: 10.1524/anly.1990.10.4.373. 
[7] J. Connor, "R-type summability methods, Cauchy criterion, P-sets and statistical convergence", Proceedings of the American mathematical society, vol. 115, no. 2. pp. 319-327, Jun. 1992, doi: 10.2307/2159248.

[8] J. Connor, "A topological and functional analytic approach to statistical convergence", in Analysis of divergence, W. Bray and C. Stanojević, Eds. Boston: Birkhäuser, pp. 403-413, 1999, doi: 10.1007/978-1-4612-2236-1_23.

[9] J. Connor and J. Kline, "On statistical limit points and the consistency of statistical convergence", Journal of mathematical analysis and applications, vol. 197, no. 2 pp. 393-399, Jan. 1996, doi: 10.1006/jmaa.1996.0027.

[10] O. Duman and C. Orhan, " $\mu$-statistically convergent function sequences", Czechoslovak mathematical journal, vol. 54, no. 2, pp. 413-422, Jun. 2004, doi: 10.1023/B:CMAJ.0000042380.31622.39.

[11] H. Fast, "Sur la convergence statistique", Colloquium mathematicae, vol. 2, no. 3-4 pp. 241-244, 1951. [On line]. Available: https://bit.ly/2M5zmod

[12] J. Fridy, "On statistical convergence”, Analysis, vol. 5, no. 4, pp. 301-313, 1985, doi: 10.1524/anly.1985.5.4.301.

[13] B. Lafuerza, J. Rodríguez and C. Sempi, "Some classes of probabilistic normed spaces", Rendiconti di matematica, vol. 17. no. 7, pp. 237-252, 1997. [On line]. Available: https://bit.ly/2sxBC00

[14] P. Harikrishnan, B. Lafuerza and K. Ravindran, "Compactness and Dboundedness in Menger's 2-probabilistic normed spaces", Filomat, vol. 30, no. 5, pp. 1263-1272, 2016. [On line]. https://bit.ly/2S1XFYb

[15] S. Karakus, "Statistical convergence on probalistic normed spaces", Mathematical communications, vol. 12, no. 1 pp. 11-23, 2007. [On line]. Available: https://bit.ly/2YVhZM2

[16] E. Kolk, "Convergence-preserving function sequences and uniform convergence", Journal of mathematical analysis and applications, vol. 238, no. 2, pp. 599-603, Oct. 1999, doi: 10.1006/jmaa.1999.6533.

[17] M. Mursaleen, Applied summability methods, Cham: Springer, 2014, doi: 10.1007/978-3-319-04609-9.

[18] T. Šalát, “On statistically convergent sequences of real numbers", Mathematica slovaca, vol. 30, no. 2, pp. 139-150, 1980. [On line]. Available: https://bit.ly/2Q87DVp

[19] B. Schweizer and A. Sklar, "Statistical metric spaces", Pacific journal of mathematics, vol. 10, n. 1, pp. 313-334, Sep. 1960. [On line]. Available: https://bit.ly/2sFiQ7q

[20] B. Schweizer and A. Sklar, Probabilistic metric spaces, New York, NY: Dover, 1983. 
[21] A. Šerstnev, "On the concept of a stochastic normalized space", Doklady akademii nauk, vol. 142, no. 2, pp. 280-283, 1963.

[22] H. Steinhaus, "Sur la convergence ordinaire et la convergence asymptotique", Colloquium mathematicum, vol. 2, pp. 73-74, 1951.

[23] B. Tripathy and R. Goswami, "On triple difference sequences of real numbers in probabilistic normed spaces", Proyecciones (Antofagasta, On line), vol. 33, no. 2, pp. 157-174, Mar. 2014, doi: 10.4067/S071609172014000200003.

[24] B. Tripathy and R. Goswami, "Multiple sequences in probabilistic normed spaces", Afrika matematika, vol. 26, no. 5-6, pp. 753-760, Sep. 2015, doi:10.1007/s13370-014-0243-1.

[25] B. Tripathy. and R. Goswami, "Fuzzy real valued p-absolutely summable multiple sequences in probabilistic normed spaces", Afrika matematika, vol. 26, no. 7-8, pp. 1281-1289, Dec. 2015, doi: 10.1007/s13370-0140280-9.

[26] B. Tripathy and R. Goswami, "Statistically convergent multiple sequences in probabilistic normed spaces", Scientific Bulletin - "Politehnica" University of Bucharest. Series A, Applied mathematics and physics, vol. 78, no. 4, pp. 83-94, 2016. [On line]. Available: https://bit.ly/2Er9Vt1

[27] B. Tripathy and M. Sen, "On generalized statistically convergent sequences", Indian journal of pure \& applied mathematics, vol. 32, no. 11, pp. 1689-1694, Nov. 2001. [On line]. Available: https://bit.ly/2PW6uQA

[28] B. Tripathy, M. Sen and S. Nath, "I-convergence in probabilistic nnormed space", Soft computing, vol. 16, no. 6, pp. 1021-1027, Jun. 2012, doi: 10.1007/s00500-011-0799-8.

[29] W. Wilczyński, "Statistical convergence of sequences of functions", Real analysis exchange, vol. 25, pp. 49-50, 2000. 